\title{
XXXVIII. A note on Mr. Burch's method of drawing hyperbolas
}

\section{F. L. O. Wadsworth E.M. M.E.}

To cite this article: F. L. O. Wadsworth E.M. M.E. (1896) XXXVIII. A note on Mr. Burch's method of drawing hyperbolas, Philosophical Magazine Series 5, 41:251, 372-378, DOI: $10.1080 / 14786449608620853$

To link to this article: http://dx.doi.org/10.1080/14786449608620853

册 Published online: 08 May 2009.

Submit your article to this journal $₫$

Q View related articles $₫$ 
Dividing the first by the second we have

from which

$$
\frac{\mathrm{D}_{1}{ }^{2}}{\mathrm{D}_{2}{ }^{2}}=\frac{1+\cos \phi}{1-\cos \phi}
$$

$$
\cos \phi=\frac{\mathrm{D}_{1}^{2}-\mathrm{D}_{2}^{2}}{\mathrm{D}_{1}^{2}+\mathrm{D}_{2}^{2}} .
$$

The reflexion is examined in a telescope with micrometer eyepiece, having two separate scales so that the lines on these scales may be made perpendicular to the long and short diameters of the ellipse. It makes no difference what the scaledivisions are, if they are alike on both scales.

When the ellipse becomes a straight line, $\mathrm{D}_{2}{ }^{2}=0$ and $\cos \phi=1$, from which $\phi=0$, or the currents are in phase. If the ellipse becomes a circle, $\mathrm{D}_{1}{ }^{2}=\mathrm{D}_{2}{ }^{2}$ and the numerator becomes 0 , consequently $\cos \phi=0$, and $\phi=90^{\circ}$, or the currents are in quadrature.

The amount of self-induction in the apparatus itself is inappreciable, and the loops keep well in time with the current. Even a considerable variation in period does not hinder the vibrations, which in this case are forced. This is possible since the mirrors, while moving very slightly, still vibrate sufficiently for telescopic observation.

Thus we have overcome the two great difficulties of many forms of this apparatus, namely, self-induction and the inability of the vibrating wires to follow a change of period. And with these objectionable features eliminated and a method of finding the exact value of $\cos \phi$, we have a practical means of determining the difference of phase.

Worcester Polytechnic Institute, Worcester, Mass.

XXXVIII. A Note on Mr. Burch's Method of Drawing Hyperbolas. By F. L. O. W ADSWORTH, E.M., M.E., Assistant Professor of Physics, University of Clicago*.

I $N$ the January number of the Philosophical Magazine $1 \mathrm{Mr}$. Burch describes a very simple and convenient method of drawing an hyperbola by the use of two similar triangles. This method is very similar to one which $I$ have been using for some time and which I have described in my lectures for the past two years, although $I$ have never published it. Mr. Burch's invention of the method antedates

* Communicated by the Author. 
mine, however, by several years, as he states that he first used it in 1885 , while it first occurred to me in 1893 . In the present note I only wish to call attention to the fact that the particular construction described is only one example of a general class of solutions of this character, and to describe two or three others which are, I think, equally simple and convenient.

In general, if in any two similar triangles two dissimilar sides are kept constant and the other sides varied, it is evident that these two varying sides, which are proportional to the two fixed sides, will be asymptotic coordinates of an hyperbola of which the modulus is the product of the two constant sides. The simplicity of the corresponding graphical or mechanical tracing of the curve depends simply upon our choice of triangles and choice of sides. In the method of construction which I most frequently employ, the two similar triangles have a common vertex at the origin $o$, fig. 1 , and the two

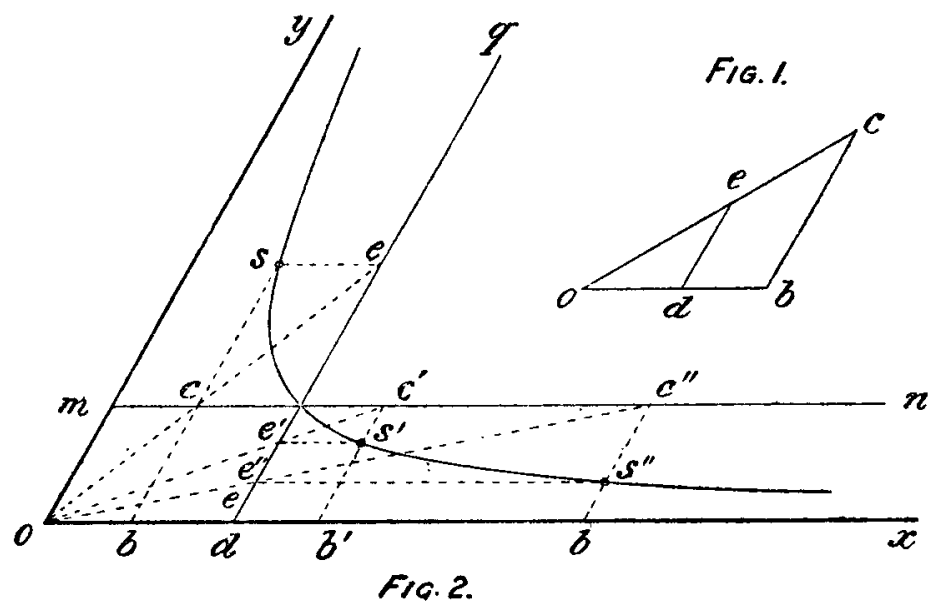

sides $o b, b c$ and $o d$, de parallel to the asymptotes of the required hyperbola. Then if we put

we have at once

$$
\begin{aligned}
& o b=x, \\
& d e=y, \\
& b c=1,
\end{aligned}
$$

$$
x y=o d \text {. }
$$

Hence if we draw a series of triangles in each of which $b c$ 
is unity and $o d$ is constant and equal to $\frac{a^{2}+b^{2}}{4}$, the sides $o b$, $d e$, of any pair represent coincident values of $x$ and $y$ in the corresponding hyperbola. These coincident values laid off along their respective axes give a series of points on the curve from which it is easy to trace the curve itself. In practice, the whole operation may be rapidly and easily performed by means of a T-square and a single triangle of which one angle is equal to the angle yox between the axes *. First draw the line $m n$ (fig. 2) parallel to and at unit distance from the axis of $x$, and the line $d q$ parallel to the axis of $y$ and at a distance from it equal to $\frac{a^{2}+b^{2}}{4}$. Then to the points $e c^{\prime} c^{\prime \prime}$ on the first line draw the lines $o c o c^{\prime} o c^{\prime \prime} \& c$. by the aid of the edge of the T-square or an auxiliary ruler, and the lines $c b, c^{\prime} b^{\prime}, c^{\prime \prime} b^{\prime \prime}$ by means of the triangle or bevel-gange. Project the points of intersection $e, e^{\prime}, e^{\prime \prime}$ of the first set of lines with the line $d q$ upon the second set of lines, giving us the points $s, s^{\prime}, s^{\prime \prime}$ on the required hyperbola. This method is particularly rapid and convenient in plotting rectangular hyperbolas on cross-section paper, the only instrument then necessary being a rule to draw the radial lines $o c, o c^{\prime}, \& c$.

If desired, an instrument can easily be constructed on these lines to trace the curve mechanically, but generally the graphical process is more rapid. A whole series of contours to the thermodynamic surface ( $p v=$ const.) can be drawn by this process in a very few minutes, the same set of lines oc, $o c^{\prime}, o c^{\prime \prime}$, and $c b, c^{\prime} b^{\prime}, c^{\prime \prime} b^{\prime \prime}$, answering for all the curves.

2nd method.-Make the vertices of the two similar triangles coincide at $c$ instead of $o$ as before, and make $a b=o c=x$, and $c d=y$ (fig. 3). Take a point $a$ on the axis of $y$ at unit distance from the origin and draw from it the lines $a c, a c^{\prime}, a c^{\prime \prime}$ to points on the axis of $x$, and the lines $b c, b^{\prime} c^{\prime}, b^{\prime \prime} c^{\prime \prime}$ parallel to the $y$ axis as before. Mark off a distance equal to $f d=\frac{a^{2}+b^{2}}{4}$ on the edge of a triangle (or bevel-gauge), $\mathrm{Q}$, of which the angle at $d$ is equal to the angle yox, and slide this triangle along each of the lines $b c, b^{\prime} c^{\prime}, b^{\prime \prime} c^{\prime \prime}$, \&c., until the point $f$ intersects the corresponding line $a c, a c^{\prime}$, or $a c^{\prime \prime}$. The points $d, d^{\prime}, d^{\prime \prime}$ will then evidently be points on the hyperbola. In practice it is not necessary to draw the lines $b c, b^{\prime} c^{\prime}, \& c$. at all, it suffices to place a T-square whose blade is parallel to the axis of $y$, so that its edge passes through any of the

* It is convenient to use for this purpose an ordinary steel bevelgauge the two blades of which may be adjusted to the required angle. 
points on the axis of $x$; slide the triangle along this edge until the point $f$ falls on the corresponding line from $a$, and

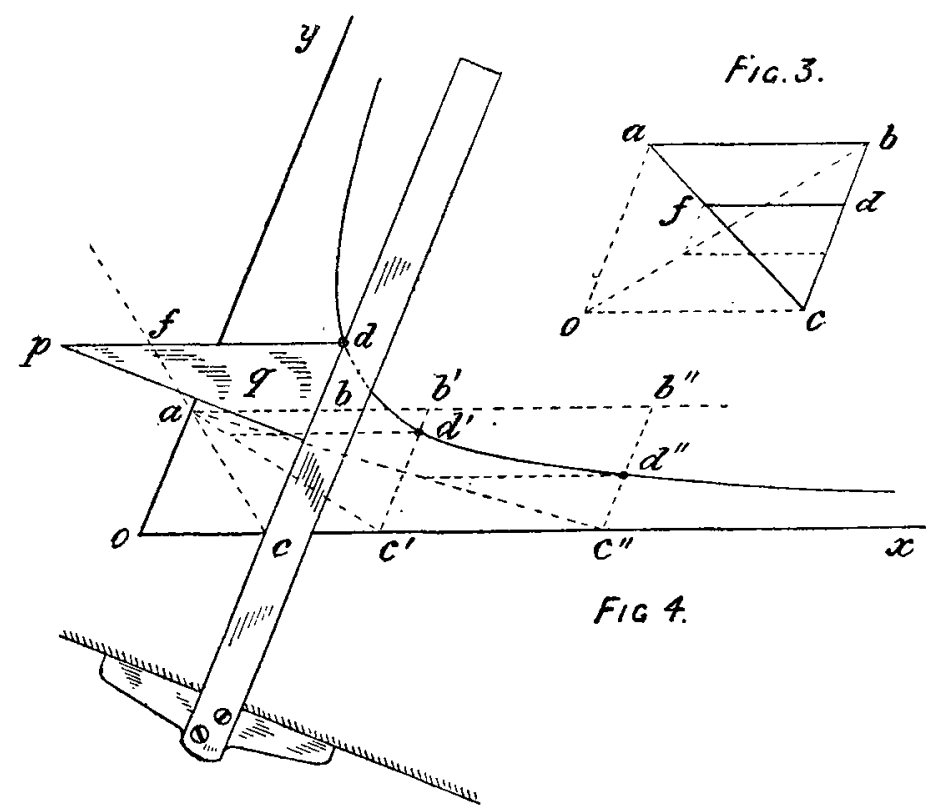

mark the position of the vertex $d$ of the triangle (see fig. 4). This method is perhaps even more rapid and convenient than the first, as it involves the drawing of only one set of lines $a c, a c^{\prime}, \& c$. Like the first, it involves the use of only a triangle and a T-square.

$3 r d$ method.-Let the vertices of the two similar triangles coincide at $b$ (fig. 5). Then if $b c=$ unity we have as before

$$
x y=f b \text {. }
$$

To determine the points on the curve graphically in this case, we need a triangle or bevel-gauge of an angle equal to $y o x$ and a parallel ruler. The side $b c$ of the triangle or bevelgauge should be of unit length and a distance $f b=\frac{a^{2}+b^{2}}{4}$ laid off on the side $b q$. The triangle is placed with its side $b q$ coinciding with the axis of $x$, and one edge of the parallel ruler is brought against the point $c$ of the triangle and a pin placed at the origin. The other blade of the parallel ruler is then moved out until it passes through the point $f$ on the 
horizontal edge of the triangle, as in fig. 6 . The point $e$ at which the blade of the ruler intersects the side $b c$ of the

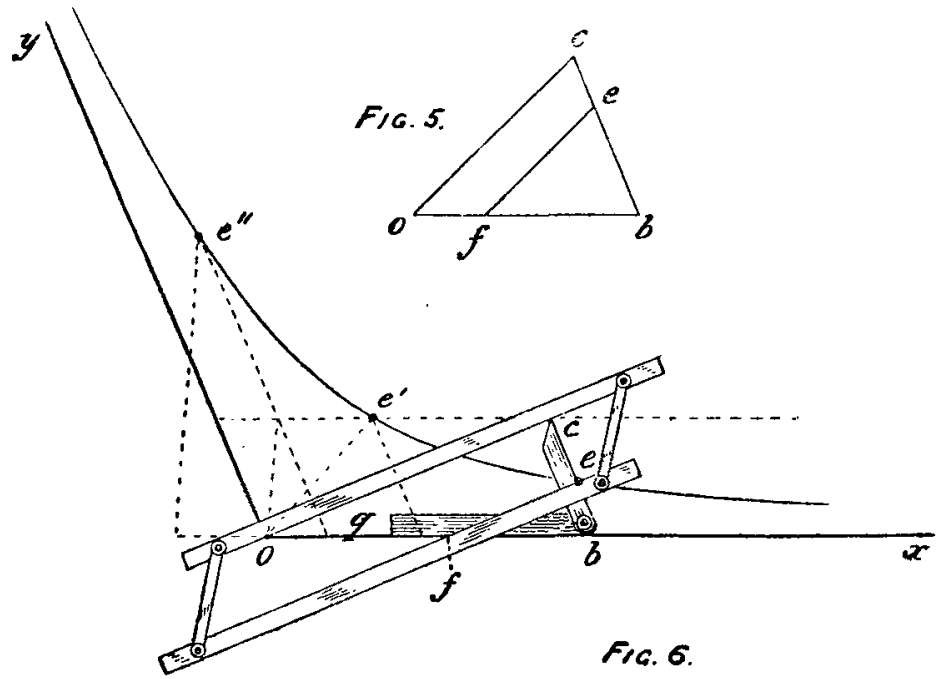

triangle will be one of the points on the required curve, the others of which may be found by sliding the triangle along the axis of $x$, always keeping the two edges of the parallel ruler in contact with the three points $o, c$, and $f$. This method is somewhat simpler mechanically, but not quite so rapid and convenient as either of the preceding.

It is evident that there are six other possible solutions to be obtained by combining the sides ac, ae (fig. 1) with each pair of adjacent sides. But these solutions are unsatisfactory graphically, because simultaneous values of $x$ and $y$ will be represented by lines inclined to each other at an angle different from the angle between the axes. If the angle at $a$ is made equal to the angle between the axes, we have one of the three solutions already considered.

The use of two similar triangles in the graphical, and more particularly the mechanical tracing of curves, is of wide application. By their aid we may always express the product or quotient of two variable quantities geometrically as the length (tensor) of one of the sides. Other applications of this principle will be found in a recent paper of the anthor's on the mountings of concave grating spectroscopes*.

" "Fixed Arm Concave Grating Spectroscopes," F. L. O. Wadsworth. Astro-Physical Journal, rol. ii. p. 370 (I)ec. 1895). 
Mr. Burch's Method of Drawing Hyperbolas.

The Use of the Quadruplane as a Hyperbolagraph.-The two asymptotic coordinates of any hyperbola evidently form two sides of a parallelogram of constant area. Hence any hyperbola can be readily traced by the use of the SylvesterKempe quadruplane linkage, the four vertices of which lie at the four angular points of a parallelogram of constant area and constant obliquity*.

The product of the adjacent sides of this parallelogram, or as Sylvester calls it the "modulus" of the cell, is equal to

$$
\mathrm{B}^{2}-\mathrm{A}^{2} \frac{\sin \alpha_{1} \sin \alpha_{2}}{\sin ^{2} \theta}=\mathrm{M} \text {, }
$$

where $B$ and $A$ are the distances between the pivolal points of the long and short links of the cell, $\alpha_{1}$ and $\alpha_{2}$ the angles adjacent to the line joining the pivotal points, and $\theta$ the angle subtended by this line at the intervening vertex. If we make each link of the cell symmetrical we have

and

$$
\begin{aligned}
& \alpha_{1}=\alpha_{2}=90-\theta / 2, \\
& \therefore M=\frac{B^{2}-A^{2}}{4 \sin ^{2} \theta / 2} .
\end{aligned}
$$

The obliquity of the parallelogram is equal to $\theta$.

Hence to describe an hyperbola whose axes are $a$ and $b$ we must make the modulus of the cell equal to the modulus of the hyperbola, or

$$
\mathrm{M}=\frac{a^{2}+b^{2}}{4},
$$

and the angle $\theta$ equal to the angle between the asymptotes, or

$$
\theta=\sin ^{-1} \frac{2 a b}{a^{2}+b^{2}} \text {. }
$$

Then if one vertex of the cell is fixed and an adjoining vertex is moved along a straight line (the edge of a T-square or straight edge for example), passing through the fixed vertex, the vertex diagonally opposite the latter will describe the required hyperbola, having the fixed point as origin and the straight line as one of the asymptotes. To describe different hyperbolas it is necessary to be able to vary both $\theta$ and M. 'The first may be easily done by making each link in two halves, pivoted together at the vertex of the link with a divided sector and clamp, by means of which the desired

"See "The History of the Plagiograph," Sylvester, ' Nature,' vol. xii. p. 214 ; also Kempe, 'Lecture on Linkages,' p. 25 et seg. 
angle $\theta$ may be laid off. The modulus of the cell is best changed by varying either A or B. This may be conveniently done by making each of either the long pair or the short pair of links like the legs of a pair of proportional dividers.

A small model of an instrument on the above lines has been constructed and found to work very easily and accurately. Like all hyperbolagraphs, however, the range of motion is limited (although larger in this than in most forms), and a considerable amount of time is necessary for the preliminary adjustment. For these reasons I have generally found one of the preceding graphical solutions more rapid and convenient, especially when a number of curves are to be drawn on the same sheet.

This application of the quadruplane, which occurred to me recently while making an application of the Peaucellier linkage to a concave grating mounting *, seems so simple and obvious that I feel sure it must have occurred to others as well as myself; but as I have not been able to find any suggestion to this effect in any of the papers on the subject that I have examined, I have ventured to present the foregoing description as another illustration of the practical application of the beautiful geometrical discovery of Prof. Sylvester and Mr. Kempe.

Ryerson Physical Laboratory,

University of Chicago, U.S.A., January 1896.

XXXIX. A Duplex Mercurial Air-Pump.

$B y$ R. W. Woon †.

IN working with highly exhausted tubes, such as are used for the production of the Röntgen rays, one of the difficuities met with is the speedy deterioration of the vacuum due to the liberation of gas from the electrodes and the glass. If the tube be thoroughly heated, while on the air-pump, this trouble is partially remedied, but even with this precaution the tubes are not very durable and have to be pumped out frequently. In order to overcome this difficulty $I$ have constructed a new form of mercurial air-pump, which can be made on a very small scale and attached permanently to the Röntgen tube. By this arrangement, any traces of gas that

* "On the Use and Mounting of the Concave Grating as an Analyzing or Direct Comparison Spectroscope," The Astro-Physical Journal,' vol. iii. p. 47 (Jan. 1896).

+ Communicated by the Author. 